\title{
Oral glucose tolerance test in patients with undiagnosed diabetes and coronary artery disease: When should it be performed?
}

\author{
A. Kollias • E. Diamanti-Kandarakis
}

Received: 20 November 2011 / Accepted: 5 January 2012 /Published online: 4 February 2012

(C) Springer-Verlag 2012

Keywords Coronary artery disease $\cdot$ Diabetes ·

Oral glucose tolerance test

\begin{abstract}
Abbreviations
AMI Acute myocardial infarction

CAD Coronary artery disease
\end{abstract}

To the Editor: We read with great interest the study by Doerr et al. [1] showing that in patients with undiagnosed diabetes who were subjected to coronary angiography, abnormal glucose regulation was documented in $49 \%$, according to the results of an OGTT. Moreover, $\mathrm{HbA}_{1 \mathrm{c}}$ values appeared to be inferior in terms of sensitivity in recognising patients with silent diabetes [1]. These results are in line with the current literature, showing that in patients with coronary artery disease (CAD), previously undiagnosed abnormal glucose regulation (impaired glucose tolerance or diabetes) is highly prevalent, with rates exceeding $50 \%$ on occasion $[2,3]$. Most importantly, the prevalence of abnormal glucose regulation seems to be similar for acute and elective hospital admissions of patients with CAD [3]. These studies provide evidence that OGTT should be part of the assessment of all patients with CAD and unknown diabetes.

\footnotetext{
A. Kollias $(\bowtie) \cdot$ E. Diamanti-Kandarakis

Third University Department of Internal Medicine,

Sotiria Hospital,

152 Mesogion Avenue,

Athens 11527, Greece

e-mail: taskollias@gmail.com
}

However, there is uncertainty as to when OGTT should be performed. Doerr et al. reported that the OGTT was undertaken on the day after the coronary angiography and stated that a limitation of the study was that OGTT was not performed before angiography [1]. Two questions arise: what is the reproducibility of OGTT in general and is this worsened during acute coronary events or elective diagnostic coronary procedures? Previous studies have shown that OGTT has a moderate reproducibility especially in individuals with impaired glucose tolerance [4]. The reproducibility seems to be even lower in patients with acute coronary events. Jiménez-Navarro et al. reported that in patients admitted with acute coronary syndromes the correlation between the OGTT performed the day after coronary revascularisation and the OGTT 1 month later was poor (weighted $\kappa 0.23 \pm 0.1$ ) [5]. Bronisz et al. also showed that the prevalence of disturbances in glucose metabolism as assessed by OGTT at 3 months was significantly lower than at discharge (29\% vs. $48 \%$ ) for patients with acute myocardial infarction (AMI), implying that the dysregulation in glucose metabolism observed in such patients may be transient [6]. On the other hand, Norhammer et al. suggested that the rates of abnormal glucose regulation (based on OGTT) in patients with AMI were similar prior to discharge and 3 months after discharge (although the degree of concordance was not reported) [7].

Acute coronary events are known to trigger inflammatory responses which in turn are associated with insulin resistance, but whether this has an impact on glucose tolerance status has not been well studied. Moreover, there are no data concerning the potential mechanisms through which acute coronary events or even elective coronary angiography in stable patients affect glucose tolerance status. Goldberg et al. 
showed that uncomplicated diagnostic coronary angiography induces a systemic inflammatory response in patients with stable angina [8]. More specifically, there was a significant increase in C-reactive protein and interleukin-6 levels at the first $24 \mathrm{~h}$ in both the coronary angiography and percutaneous coronary intervention groups [8]. Thus, a transient insulin resistance state may be expected during such events or procedures.

In conclusion, the timing of the OGTT in relation to preceding events (angiography or AMI) should be taken into account before safe conclusions can be drawn regarding the prevalence of abnormal glucose regulation. Until new evidence accumulates, it would be wise to suggest that the diagnosis of impaired glucose tolerance or diabetes based on OGTT be delayed until patients with $\mathrm{CAD}$ are in a stable condition, preferably after hospital discharge.

Duality of interest statement The authors declare that there is no duality of interest associated with this manuscript.

Contribution statement $\mathrm{AK}$ and EDK were responsible for the conception of the letter; AK drafted the article and EDK revised it critically. All authors approved the final version.

\section{References}

1. Doerr R, Hoffmann U, Otter W et al (2011) Oral glucose tolerance test and $\mathrm{HbA}_{1 \mathrm{c}}$ for diagnosis of diabetes in patients undergoing coronary angiography the Silent Diabetes Study. Diabetologia 54:2923-2930, Erratum 54: 2968

2. Bartnik M, Rydén L, Ferrari R et al (2004) The prevalence of abnormal glucose regulation in patients with coronary artery disease across Europe. The Euro Heart Survey on diabetes and the heart. Eur Heart J 25:1880-1890

3. Hu DY, Pan CY, Yu JM, China Heart Survey Group (2006) The relationship between coronary artery disease and abnormal glucose regulation in China: the China Heart Survey. Eur Heart J 27:2573-2579

4. Mooy JM, Grootenhuis PA, de Vries H et al (1996) Intra-individual variation of glucose, specific insulin and proinsulin concentrations measured by two oral glucose tolerance tests in a general Caucasian population: the Hoorn Study. Diabetologia 39:298-305

5. Jiménez-Navarro MF, Garcia-Pinilla JM, Garrido-Sanchez L et al (2010) Poor reproducibility of the oral glucose tolerance test in the diagnosis of diabetes during percutaneous coronary intervention. Int J Cardiol 142:245-249

6. Bronisz A, Kozinski M, Magielski P et al (2011) Value of oral glucose tolerance test in the acute phase of myocardial infarction. Cardiovasc Diabetol 10:21

7. Norhammer A, Tenerz A, Nilsson G et al (2002) Glucose metabolism in patients with acute myocardial infarction and no previous diagnosis of diabetes mellitus: a prospective study. Lancet 359:2140-2144

8. Goldberg A, Zinder O, Zdorovyak A et al (2003) Diagnostic coronary angiography induces a systemic inflammatory response in patients with stable angina. Am Heart J 146:819-823 